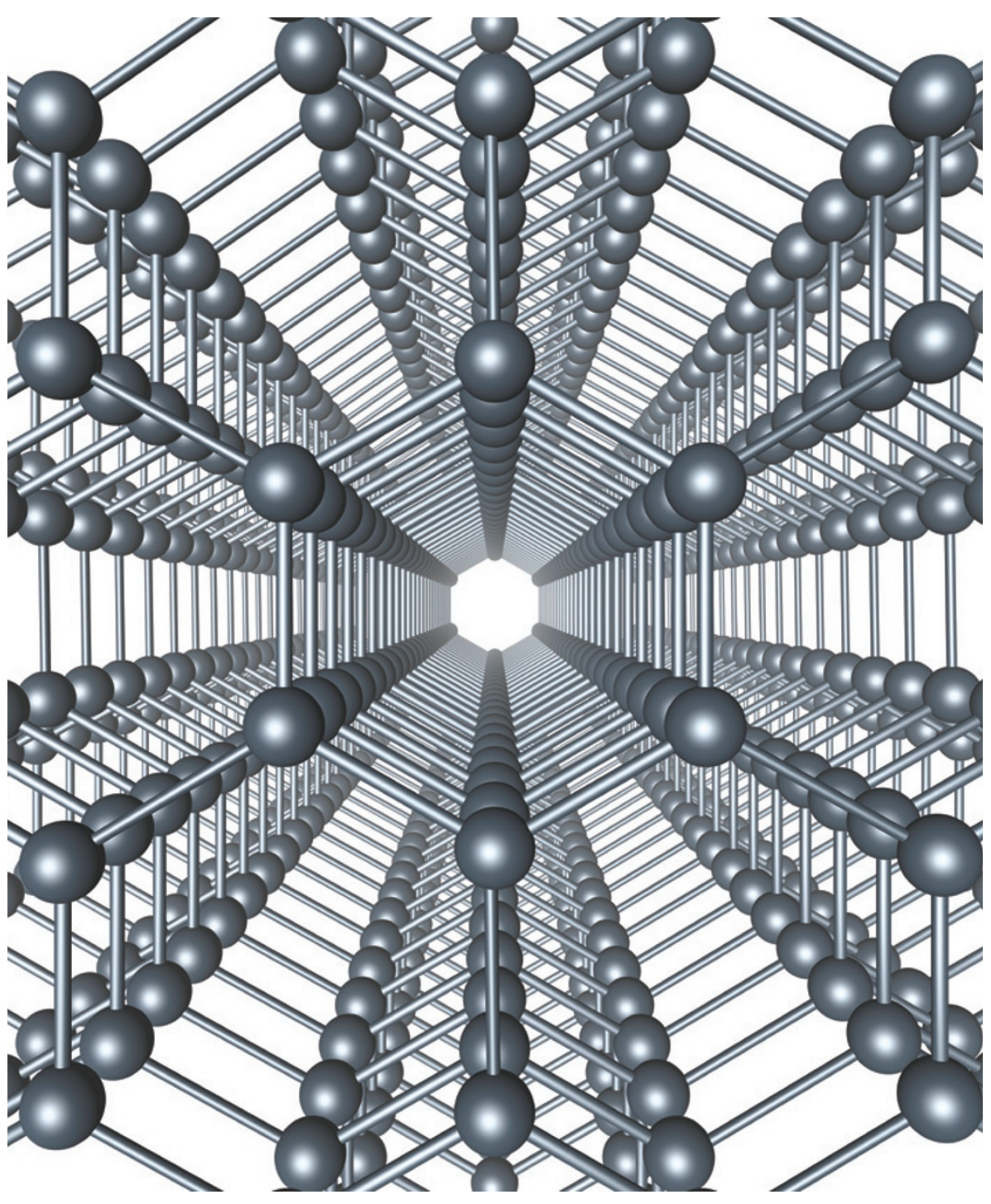

MATERIALS SCIENCE

Super carbon

Graphene is phenomenally strong, thin, flexible,
transparent and conductive - and applications beckon.

BY NEIL SAVAGE

$\mathrm{O}$ ne day in 2004, Andre Geim and Konstantin Novoselov, two chemists at the University of Manchester in the United Kingdom, peeled down graphite to a speck of graphene and ran electrons through it. In doing so they opened up a world of possibilities based on this material's remarkable properties - and won themselves the 2010 Nobel Prize in Physics.

Though only recently identified as a distinct form of carbon, graphene is not exotic. Layers of it stacked together form graphite, the 'lead' in pencils. Graphene rolled up becomes a carbon nanotube. Wrapped into a sphere, it's a fullerene or, colloquially, a 'buckyball'.

Unlike other carbon forms, graphene is a two-dimensional material, and the first example of such a thing in the real world. Philip Russell Wallace, a theoretical physicist at McGill University in Montreal, Canada, predicted the electronic structure of graphene back in 1947, but many scientists believed 2D crystals were too unstable to exist. What Geim and Novoselov managed to pull off, through their savvy use of sticky tape, was a sheet of carbon a few micrometres across and just a single atom thick. This is because in graphite the atomic bonds between the layers are much weaker than the bonds across its layers.

Graphene's arrangement gives rise to some astonishing properties. Electrons move through it as if they have no mass. The material boasts an electron mobility (a measure of how fast charges travel) 100 times that of silicon. At room temperature, electrons can travel for several micrometres through graphene without scattering - an order of magnitude further than in any other material. And researchers have demonstrated graphene transistors operating at more than twice the speed of the best silicon transistors of similar size. The behaviour of electrons in graphene also provides physicists studying quantum mechanics with a new research tool. Instead of having to use superconductors or fluids cooled to extremely low temperatures, they can observe the seemingly massless activity of electrons on their lab benches.

Graphene stands out in other ways as well. Although it is the thinnest material known to exist, it is also the strongest ever measured: 100 times stronger than steel. It conducts heat better than the previous champion, diamond. The atoms in graphene are packed so tightly that not even the smallest atom - helium - can pass through it. Yet graphene also stretches easily, giving it the ability to bend where other hardy materials would snap.

One property that graphene lacks is a bandgap - a range of energy states where electrons cannot exist. Bandgap is a crucial characteristic that enables semiconductor transistors to switch between on and off states to form the zeroes and ones of digital technology. But what's a problem for electronics is a boon for optics: graphene's lack of a bandgap means the material absorbs light at roughly the same level across the spectrum, from ultraviolet far into infrared. Exploiting graphene's optical and electronic abilities along with its strength and flexibility could lead to foldable plastic smartphones, cheaper solar cells, or sensors that can detect single molecules of gas or identify individual DNA bases.

Despite the intense research interest in graphene, real-world applications are in their infancy. It's not yet clear if it will be able to supplant silicon in transistors any time soon (see 'Back to analogue', page S34). And although carbon is ubiquitous - simply heating any organic material to high temperature in a vacuum produces plenty - engineers are still grappling with ways to make high-quality graphene in sufficient quantities and at a cost to sustain commercial applications (see 'Beyond sticky tape', page S32). Silicon took decades to find its signature role in technology; graphene's journey has only just begun.

Neil Savage is a freelance writer based in Lowell, Massachusetts. 


\section{BATTLE OF THE ALLOTROPES}

For the past 25 years, researchers have been exploring the novel properites of one form of single-layer carbon after another.

Nanotube

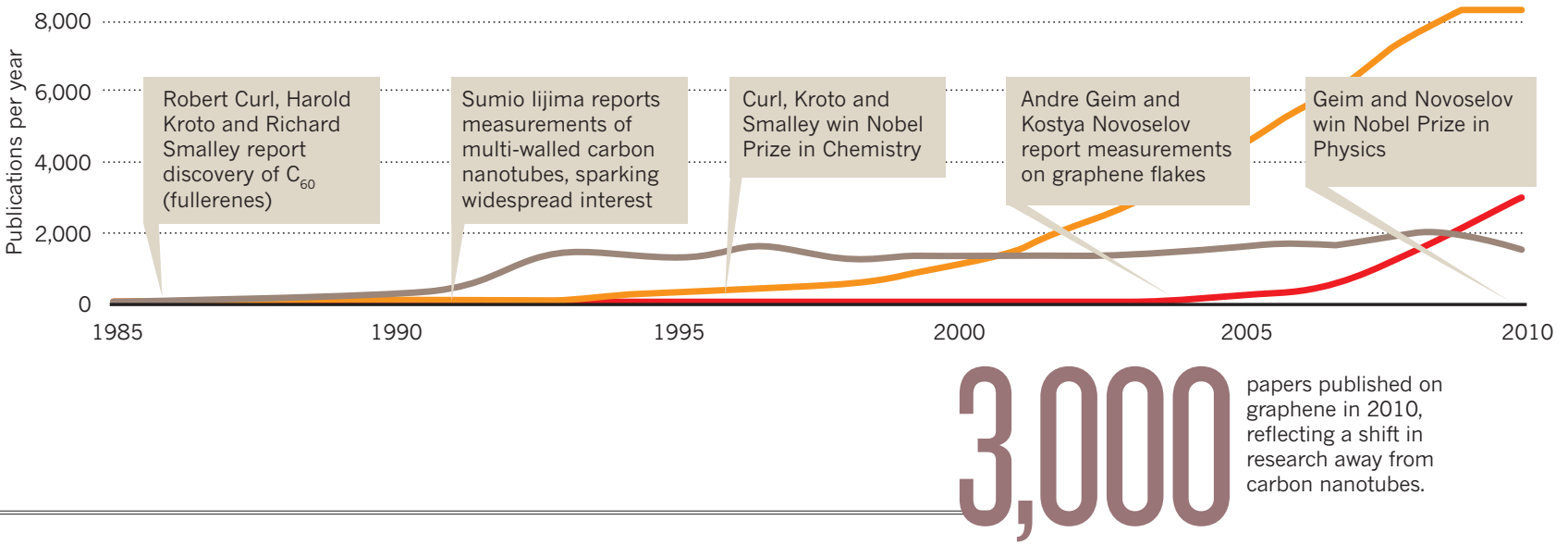

\section{SUPER-MATERIAL}

Graphene stands out for its superlative mechanical, thermal and electronic properties.

Tensile strength (MPa)

$1,000,000,000$

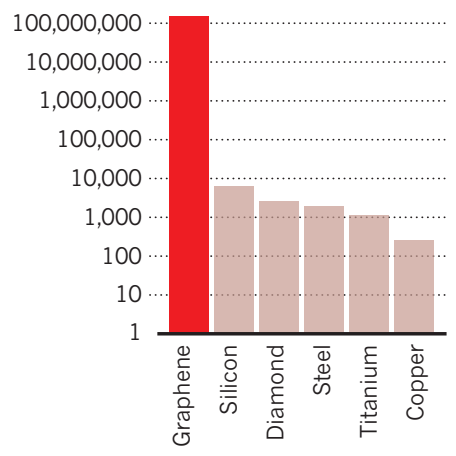

Stiffness (Young's modulus, GPa) 10,000

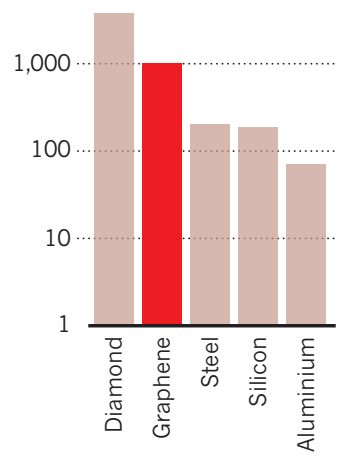

Electron mobility $\left(\mathrm{cm} \mathrm{V}^{-1} \mathrm{~s}^{-1}\right)$ $1,000,000$

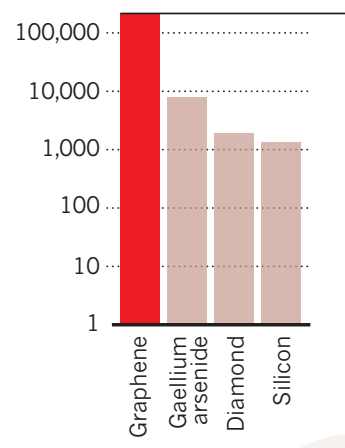

Thermal conductivity ( $\mathrm{W} \mathrm{m}^{-1} \mathrm{~K}^{-1}$ ) 10,000

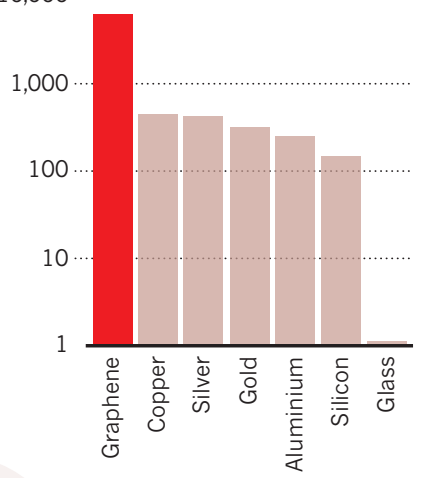

\section{EXPLOSIVE GROWTH}

As research on graphene takes off, technology development is heavily concentrated in the United States and South Korea.
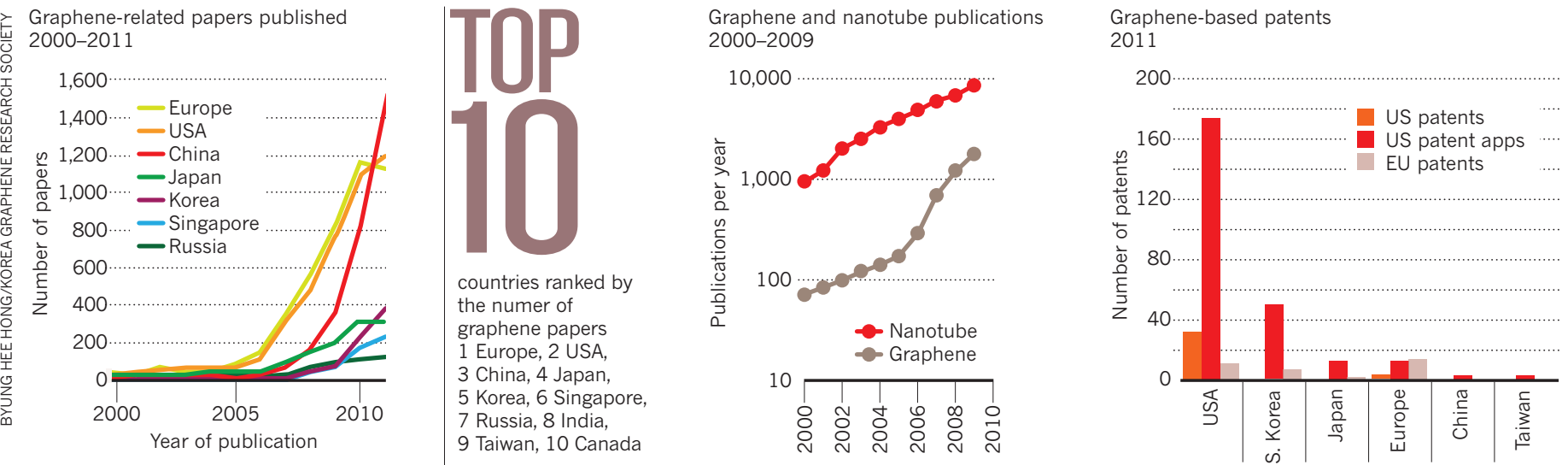